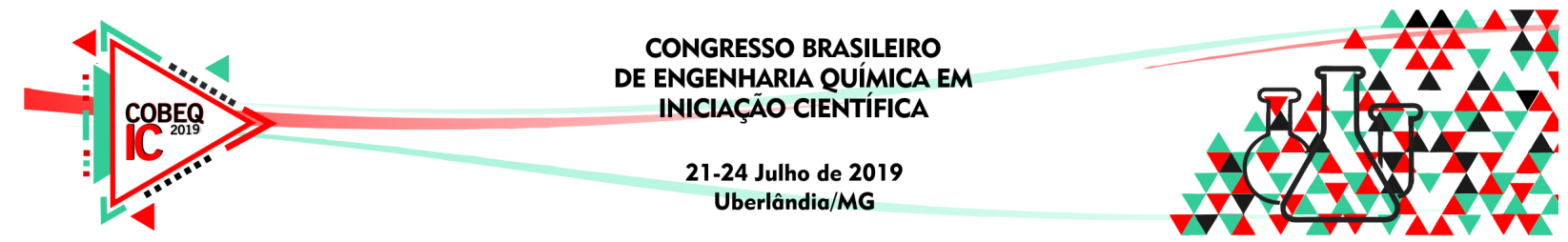

\title{
IMPACTO TÉCNICO-ECONÔMICO DE UMA GERATRIZ NÃO LINEAR EM COMPARAÇÃO A UMA ALETA PINIFORME
}

\author{
L. C. GIMENES, R. A. MALAGONI e L. G. M. VIEIRA \\ Universidade Federal de Uberlândia, Faculdade de Engenharia Química \\ E-mail para contato: gimenesluiscruz@gmail.com
}

\begin{abstract}
RESUMO - Aletas são dispositivos térmicos baseados em superfícies estendidas que majoram a transferência de calor de uma superfície para um fluido. As aletas devem ser construídas a partir de bons condutores térmicos e pequenas espessuras, a fim de garantir, simultaneamente, estruturas leves para instalação e tratamento matemático unidimensional para previsão das distribuições térmicas e taxas de dissipação de calor. Aletas no formato cilíndrico são denominadas de aletas piniformes, cuja descrição físico-matemática é bem-posta na literatura por causa da área da seção reta à condução térmica ser constante ao longo de todo o comprimento do dispositivo. Isto posto, este estudo teve como objetivo avaliar o impacto técnico e econômico de uma aleta de seção reta variável ao longo do comprimento do dispositivo. De acordo com os principais resultados, a utilização de uma geratriz não linear crescente mostrou que, nas mesmas condições operacionais das aletas piniformes (área da base, comprimento e condições térmico-fluidodinâmicas), foi possível incrementar a dissipação térmica em até $71 \%$. Por sua vez, a utilização de uma geratriz não linear decrescente impactou em uma redução de $18 \%$ na taxa de dissipação térmica. Além de a aleta de geratriz não linear crescente ter sido a mais dissipativa dentre todas, os estudos mostraram que os custos com a aquisição de metais ou ligas para a construção dela são incrementados em $14 \%$ apenas.
\end{abstract}

\section{INTRODUÇÃO}

As aletas são dispositivos térmicos utilizados para estender áreas de troca térmica entre uma superfície e um fluido, cujo efeito almejado é o aumento na taxa de dissipação de calor (Incropera e DeWitt, 2003). Assim, durante a dissipação térmica pelas aletas, há simultaneamente a presença de condução e convecção de calor (Bird et al., 2004).

No interior de uma superfície estendida, se nenhuma providência técnica for tomada, a condução de calor é bidimensional (Bennet e Myers, 2003), cujo tratamento físicomatemático deve ser feito necessariamente pela Equação da Difusão de Calor, representada pela Equação 1, com as devidas simplificações (materiais isotrópicos, transporte de calor em estado estacionário e sem taxa de geração ou consumo de calor), conforme se vislumbra na EDP representada pela Equação 2. 


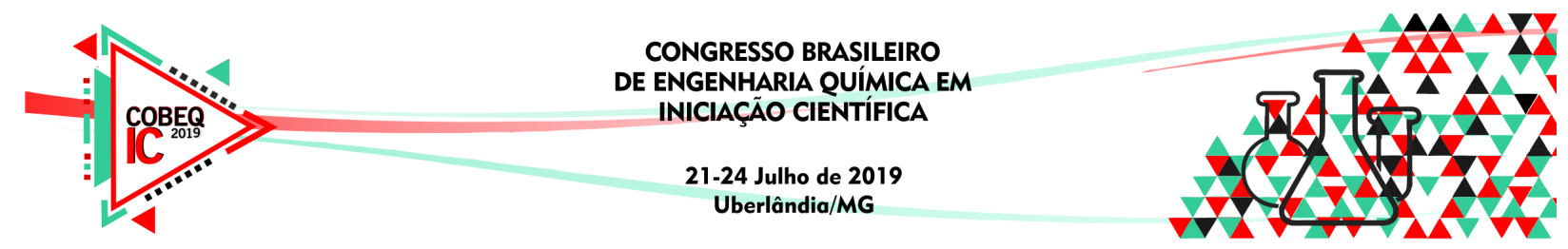

$$
\begin{aligned}
& \rho c_{P} \frac{\partial T}{\partial t}=\frac{1}{r} \frac{\partial}{\partial r}\left(k r \frac{\partial T}{\partial r}\right)+\frac{1}{r \operatorname{sen} \theta} \frac{\partial}{\partial \theta}\left(k \frac{\partial T}{\partial \theta}\right)+\frac{\partial}{\partial r}\left(k \frac{\partial T}{\partial z}\right)+G \\
& \frac{1}{r} \frac{\partial}{\partial r}\left(r \frac{\partial T}{\partial r}\right)+\frac{\partial^{2} T}{\partial r^{2}}=0
\end{aligned}
$$

Porém, se forem usados bons condutores térmicos e pequenas espessuras longitudinais nas superfícies estendidas, tais estruturas passam a ser denominadas de aletas, cuja principal característica é prover uma condução térmica unidimensional ao longo da posição axial. Em provendo uma condução unidimensional axial, a descrição físico-matemática dessa estrutura pode ser feita por um Balanço de Energia que origina uma EDO de Segunda Ordem (Araújo, 1978), representada pela Equação 3, cuja solução é mais simples em comparação à EDP (Equação 2), sem, contudo, comprometer a confiabilidade dos resultados (Zill e Cullen, 2001).

$$
\frac{d^{2} T}{d z^{2}}+\left(\frac{1}{A_{C}} \frac{d A_{c}}{d z}\right) \frac{d T}{d z}+\left(\frac{h}{k} \frac{1}{A_{C}} \frac{d A_{S}}{d z}\right)\left(T-T_{\infty}\right)=0
$$

As Condições de Contorno aplicáveis à Equação 3 são representadas pela Equação 4 e Equação 5, as quais representam a temperatura na base da aleta (equivalente à superfície que se deseja estender) e equivalência de fluxos (condutivo e convectivo) na extremidade da aleta, respectivamente.

$$
\begin{aligned}
& T(0)=T_{0} \\
& -\left.k \frac{d T}{d z}\right|_{L}=h\left(T_{L}-T_{\infty}\right)
\end{aligned}
$$

Aletas com formato cilíndricos são denominadas de aletas piniformes. As aletas piniformes são fáceis de serem construídas, bem como proporcionam área disponível à condução de calor sempre constante. Geometricamente, representam a revolução de uma reta de coeficiente angular nulo ao redor de seu eixo axial. $O$ fato de área à condução ser constante ao longo de toda a dimensão axial da aleta piniforme facilita, sobremaneira, a solução da EDO oriunda do Balanço de Energia (Equação 3). Se ao longo da posição axial houver variação crescente ou decrescente da área disponível à condução de calor, poderá haver condições mais favoráveis para aumentar a dissipação de calor pela estrutura, o que representa uma vantagem técnica almejada por muitos processos térmicos. No entanto, apesar da vantagem térmica mencionada, mesmo em se tratando de uma EDO, haverá, indubitavelmente, um aumento na complexidade numérica para prover as respectivas previsões das distribuições térmicas e das taxas de calor dissipadas. Por fim, vale mencionar que tais aletas são geometricamente geradas pela revolução de uma função matemática $f(z)$ ao redor dos respectivos eixos axiais.

Isto posto, esse estudo visou ao estudo do comportamento térmico de aletas de seção reta variável (crescente e decrescente) frente ao padrão ordinariamente utilizado nos processos térmicos (aletas piniformes). Buscou-se, especificamente, realizar uma avaliação comparativa 


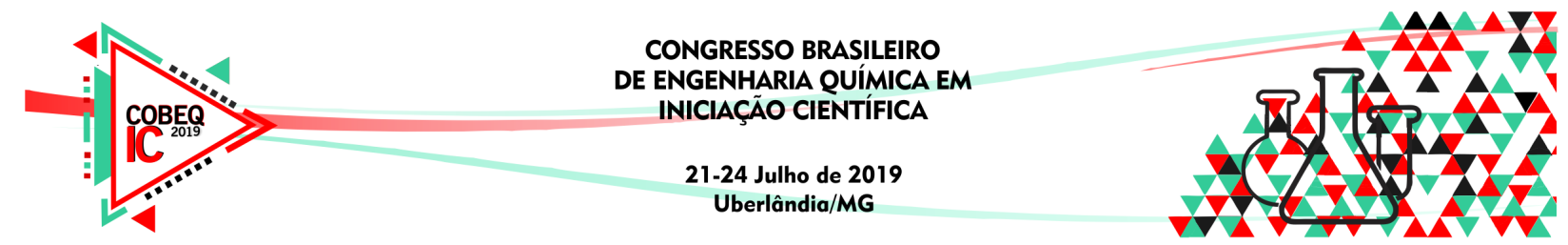

das distribuições térmicas, das taxas de dissipação de calor e do custo com material entre a aleta piniforme e suas variações.

\section{METODOLOGIA}

Foram utilizados três tipos de aletas neste estudo: uma aleta piniforme, uma aleta de seção reta variável crescente e uma aleta de seção reta variável decrescente, conforme a representação esquemática da Figura 1. Foram utilizadas geratrizes quadráticas para geração das estruturas das aletas por meio da revolução delas ao redor do eixo axial (z), cujos parâmetros podem ser vistos na Tabela 1. Independentemente do formato das aletas, todas apresentaram os mesmos comprimentos $(L=0,100 \mathrm{~m})$, diâmetros da base $(D=0,005 \mathrm{~m})$, condutividades térmicas $(k=14 \mathrm{~W} / \mathrm{mK})$ e coeficientes convectivos de transferência de calor $\left(h=5 \mathrm{~W} / \mathrm{m}^{2} \mathrm{~K}\right)$. Nos cálculos, considerou-se ainda que todas as aletas tinham a base submetida à temperatura de $150^{\circ} \mathrm{C}\left(T_{0}\right)$ e as superfícies laterais submetidas ao ar à temperatura ambiente $\left(T_{\infty}\right)$ de $20^{\circ} \mathrm{C}$.

Tabela 1 - Parâmetros das geratrizes das aletas.

\begin{tabular}{|c|c|c|}
\hline \multicolumn{3}{|c|}{$f_{M}(z)=a+b z^{2}$} \\
\hline \hline$M$ & $a(\mathrm{~m})$ & $b\left(\mathrm{~m}^{-1}\right)$ \\
\hline \hline 1 & 0,0025 & 0,05 \\
\hline 2 & 0,0025 & 0,00 \\
\hline 3 & 0,0025 & $-0,25$ \\
\hline
\end{tabular}

Figura 1 - Representação Esquemática das Aletas Analisadas.

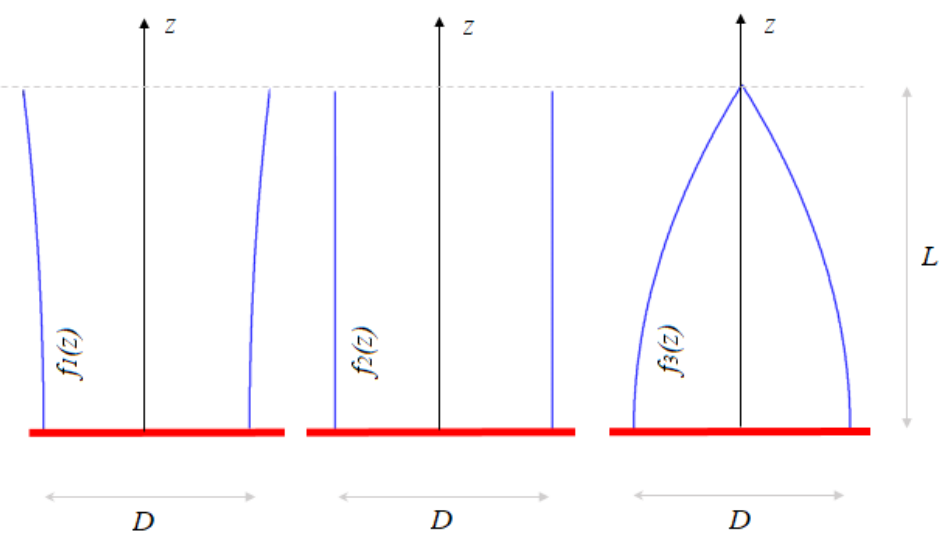

As áreas de seção reta à condução térmica $\left(A_{C}\right)$ e as áreas laterais $\left(A_{S}\right)$ disponíveis à convecção de calor, necessárias à solução numérica da Equação de Balanço (Equação 3), foram postas em função da posição axial $z$, cuja visualização pode ser dar pelas Equação 6 e Equação 7, respectivamente. O volume de cada estrutura é representado pela Equação 8.

$$
\begin{aligned}
& A_{C}=\pi\left(a+b z^{2}\right)^{2} \\
& A_{S}=2 \pi \int\left(a+b z^{2}\right) \sqrt{1+(2 b z)^{2}} d z
\end{aligned}
$$




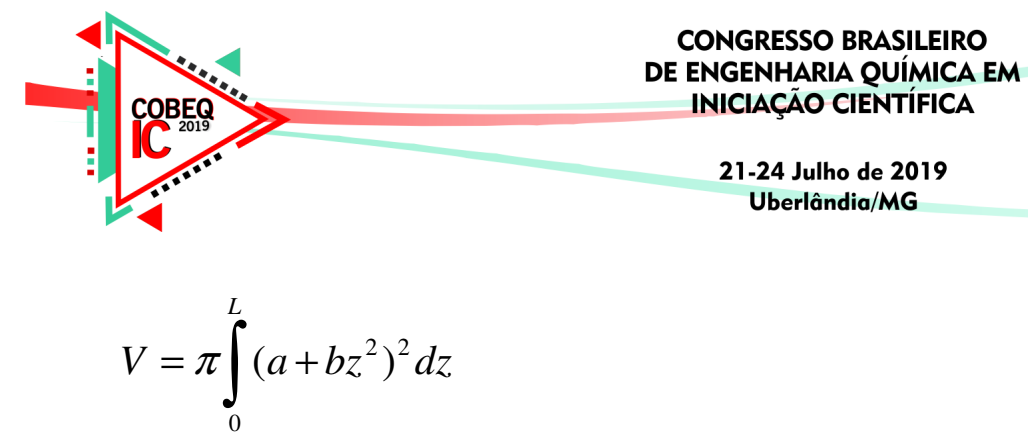

A Equação 3 foi resolvida numericamente, escolhendo-se 9 pontos de solução com passo de integração $(\delta)$ de $0,01 \mathrm{~m}$. As derivadas primeira e segunda, presentes na Equação 3 , foram aproximadas pelo método das diferenças centrais, nos termos da Equação 9 e Equação 10, respectivamente, originado, portanto, a Equação Geradora descrita na Equação 11, na qual $n$ representa cada um dos pontos do domínio em que se deseja a solução.

$$
\begin{aligned}
& \frac{d T}{d z} \cong \frac{T_{n+1}-T_{n-1}}{2 \delta} \\
& \frac{d^{2} T}{d z^{2}} \cong \frac{T_{n+1}-2 T_{n}+T_{n-1}}{\delta^{2}} \\
& {\left[\frac{1}{\delta^{2}}+\frac{2 b z}{\delta\left(a+b z^{2}\right)}\right] T_{n+1}-2\left[\frac{1}{\delta^{2}}+\frac{h}{k} \frac{\sqrt{1+(2 b z)^{2}}}{\left(a+b z^{2}\right)}\right] T_{n}+\left[\frac{1}{\delta^{2}}-\frac{2 b z}{\delta\left(a+b z^{2}\right)}\right] T_{n}=0}
\end{aligned}
$$

Por fim, as taxas de calor dissipadas pelas aletas foram calculadas pela Equação 12.

$$
q=-k\left[A_{C} \frac{d T}{d z}\right]_{z=0}
$$

\section{RESULTADOS}

As distribuições térmicas obtidas para as aletas piniforme e de seção reta variável (crescente e decrescente) podem ser visualizadas na Figura 2.

Figura 2 - Distribuições Térmicas ao longo da posição axial das aletas.

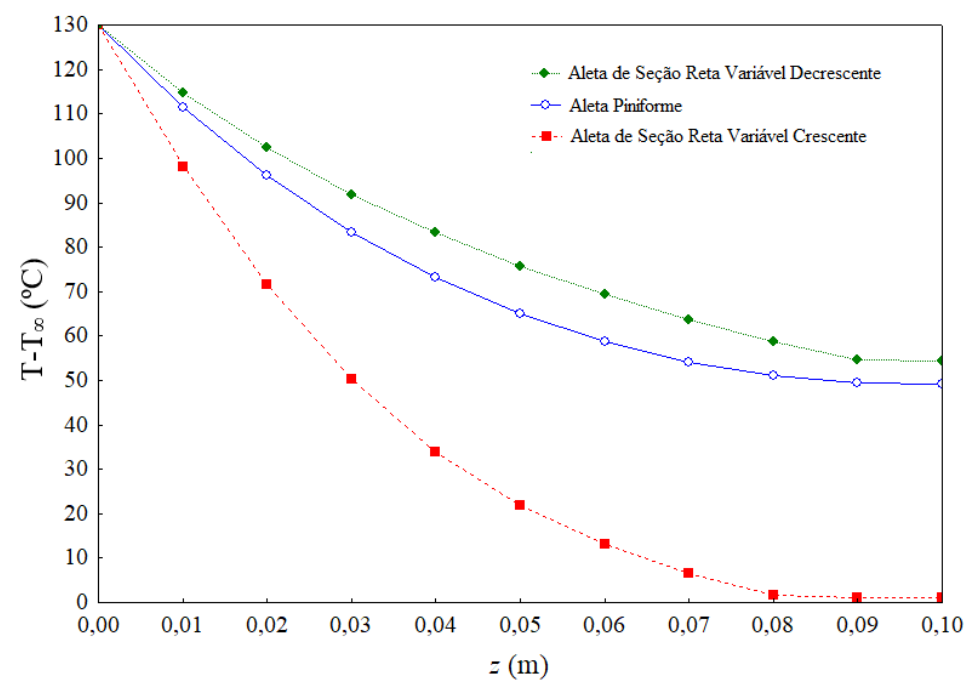




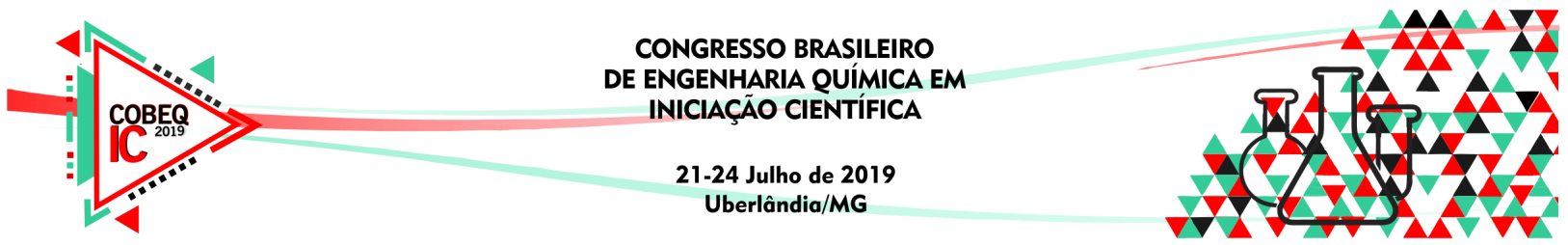

De acordo com a Figura 2, foi possível verificar que variação da área disponível à condução térmica exerceu um impacto significativo nas distribuições térmicas das aletas. Dentre as aletas analisadas, a aleta de seção reta variável crescente foi a que teve o maior decaimento térmico ao longo do eixo da aleta, enquanto que a aleta de seção reta variável decrescente foi a que apresentou o menor decaimento. Tais comportamento estão diretamente relacionados com a capacidade de cada estrutura dissipar mais ou menos calor da base para o fluido, conforme se constata na Tabela 2.

Tabela 2 - Taxas de calor dissipadas pelas aletas.

\begin{tabular}{|l|c|c|}
\hline \hline \multicolumn{1}{|c|}{ Aletas } & $q(\mathrm{~J} / \mathrm{s})$ & $V\left(\mathrm{~m}^{3}\right)$ \\
\hline \hline Seção Reta Decrescente & 0,42 & $1,03 \cdot 10^{-6}$ \\
\hline Piniforme (Padrão) & 0,51 & $1,96 \cdot 10^{-6}$ \\
\hline Seção Reta Crescente & 0,87 & $2,24 \cdot 10^{-6}$ \\
\hline
\end{tabular}

Em se tratando da capacidade de dissipação de calor pelas estruturas analisadas, a aleta de seção reta crescente apresentou a maior taxa energia dentre todas, o que, sob o ponto de vista técnico, seria a aleta mais vantajosa dentre as estruturas analisadas. De acordo com a Tabela 2, foi possível que constatar que a taxa de calor da aleta de seção reta crescente foi majorada em aproximadamente $71 \%$ em comparação à aleta piniforme, o que representa uma vantagem técnica extremamente importante nos processos de troca térmica, haja vista que essa configuração demandaria apenas um incremento na demanda de metal ou liga (massa ou custo) de aproximadamente $14 \%$. Por sua vez, a aleta de seção reta decrescente teve um decréscimo de aproximadamente $18 \%$ na taxa de dissipação de calor quando comparada à aleta padrão, o que compromete seu uso, inclusive pela dificuldade de extrusão ou usinagem de estruturas com este formato, apesar de ser uma estrutura cerca de 53\% mais leve e barata que a aleta padrão.

Outro fato interessante que também pode ser observado a partir das distribuições térmicas, refere-se ao fato das aletas conseguirem ou não alcançar o equilíbrio térmico em suas extremidades. Neste aspecto, foi possível constatar que o comprimento de $10 \mathrm{~cm}$ foi mais do que suficiente para que a aleta de seção reta variável crescente atingisse a condição de equilíbrio térmico, já se comportando, portanto, como aleta infinita com apenas $85 \%$ de comprimento disponível. Tal fato denota economia de material e uso efetivo de sua capacidade dissipativas em comparação às aletas piniforme e de seção reta decrescente.

\section{CONCLUSÃO}

O formato da aleta influenciou na capacidade de dissipação de calor pela estrutura sólida. Nas mesmas condições físico-operacionais, a aleta de seção reta crescente foi a estrutura sólida mais promissora, pois apresentou um incremento de $71 \%$ na taxa de dissipação de calor com um incremento de custo de apenas $14 \%$ na aquisição da liga metálica. Logo, o uso de geratrizes não lineares podem ser uma alternativa interessante para incrementar as eficiências dos processos térmicos, sem, contudo, onerar excessivamente os projetos com o custo de aquisição dos materiais construtivos. 


\section{NOMENCLATURA}

$A_{C}$ - área de seção reta de uma aleta (m)

$A_{S}$ - área lateral de uma aleta (m)

$c_{P}-$ calor específico do sólido que compõe a aleta $\left(\mathrm{J} / \mathrm{kg}^{\circ} \mathrm{C}\right)$

$D$ - diâmetro da base da aleta (m)

$G$ - taxa de geração ou consumo de calor no sólido $\left(\mathrm{W} / \mathrm{m}^{3}\right)$

$h$ - coeficiente convectivo de transferência de calor $\left(\mathrm{W} / \mathrm{m}^{2 \circ} \mathrm{C}\right)$

$k$ - condutividade térmica do sólido que compõe a aleta $\left(\mathrm{W} / \mathrm{m}^{\circ} \mathrm{C}\right)$

$L$ - comprimento da aleta $(\mathrm{m})$

$t$ - tempo (s)

$T$ - temperatura ao longo da posição axial da aleta $\left({ }^{\circ} \mathrm{C}\right)$

$T_{0}$ - temperatura da base da aleta $\left({ }^{\circ} \mathrm{C}\right)$

$T_{\infty}$ - temperatura do fluido no qual a aleta está inserida $\left({ }^{\circ} \mathrm{C}\right)$

$q$ - taxa de calor dissipada pela aleta (W)

$V$ - volume da aleta $\left(\mathrm{m}^{3}\right)$

$\delta$ - passo de integração (m)

$\rho$ - densidade do sólido que compõe a aleta $\left(\mathrm{kg} / \mathrm{m}^{3}\right)$

\section{NOMENCLATURA}

ARAÚJO, C. Transmissão de Calor. São Paulo: Livros Técnicos e Científicos Editora, 1978.

BENNET, C. O.; MYERS, J. E. Fenômeno de Transporte - Quantidade de Movimento, Calor e Massa. São Paulo: Editora LTC, 2003.

BIRD, R. B, STEWART, W. E., LIGHTFOOT, E. N. Transport Phenomena. São Paulo: LTC, 2004.

INCROPERA, F. P.; DEWITT, D. P. Transferência de Calor e Massa. São Paulo: LTC, 2003.

ZILL, D. G.; CULLEN, M. R. Equações Diferenciais. São Paulo: Editora Makron Books, 2001. 